\title{
Interleukin-6 expression by interactions between gynecologic cancer cells and human mesenchymal stem cells promotes epithelial-mesenchymal transition
}

\author{
KYEONG A SO ${ }^{1}$, KYUNG JIN MIN ${ }^{2}$, JIN HWA HONG ${ }^{2}$ and JAE-KWAN LEE ${ }^{2}$ \\ ${ }^{1}$ Department of Obstetrics and Gynecology, Cheil General Hospital and Women's Healthcare Center, \\ Dankook University College of Medicine, Seoul; ${ }^{2}$ Department of Obstetrics and Gynecology \\ Guro Hospital, College of Medicine, Korea University, Seoul, Republic of Korea
}

Received May 14, 2015; Accepted June 29, 2015

DOI: $10.3892 /$ ijo.2015.3122

\begin{abstract}
Epithelial-to-mesenchymal transition (EMT) facilitates the invasion and metastasis of cancer cells. EMT seems to be mediated by the interaction between cancer cells and human mesenchymal stem cells (hMSCs) in the tumor microenvironment. The present study is intended to identify specific cytokines as potent inducers of EMT associated hMSCs-tumor interactions. We used ovarian cancer cell lines (SKOV-3 and IGROV-1), endometrial cancer cell line (Ishikawa) and hMSCs (bone marrow MSC, amniotic membrane MSC and decidua MSC). The expressions of EMT markers (E-cadherin, Snail, Twist and N-cadherin) were analyzed using quantitative RT-PCR, immunofluorescence and western blot analysis. Matrix metalloproteinases (MMP-2 and MMP-9), Matrigel invasion assay, and wound healing assay were used to analyze cell migration and invasion. Gynecologic cancer cells directly co-cultured with hMSCs had contact-dependent altered morphology and growth patterns. IL-6 was elevated in all co-cultures using a human cytokine array. After IL- 6 treatment of cancer cell lines, RT-PCR and western blot analysis indicated a decrease in an epithelial marker and an increase in mesenchymal markers. Also, cancer cells with IL-6 significantly increase in MMP-2 and MMP-9 and significantly enhance the migration ability compared to untreated cells $(\mathrm{P}<0.05)$, as shown by wound healing assay. On Matrigel invasion assay, treated cells displayed significantly increased invasiveness compared to untreated cancer cells. Gyneocologic cancer cells exposed
\end{abstract}

Correspondence to: Professor Jae-Kwan Lee, Department of Obstetrics and Gynecology, Guro Hospital, College of Medicine, Korea University, 80 Guro-gu Guro-dong, Seoul 152-703, Republic of Korea

E-mail: jklee38@korea.ac.kr

Key words: interleukin-6, epithelial-mesenchymal transition, gynecologic cancer, metastasis, invasion to IL-6 acquired mesenchymal properties that facilitated metastasis and invasion by promoting EMT. The present study suggests that IL-6 of the tumor microenvironment has a critical role in oncogenic EMT.

\section{Introduction}

Epithelial cancer cells are surrounded by the tumor microenvironment, which consists of various non-transformed cells, soluble factors, signaling molecules and extracellular matrix. Interactions between tumor cells and their surrounding environment are critical for tumor growth, invasion, metastasis and therapeutic resistance (1). Tumors actively recruit cells, including mesenchymal stem cells (MSCs), and these cells may play a role in facilitating cancer progression (2).

MSCs are non-hematopoietic stem cells found in several different tissues, including umbilical cord blood, placenta, adipose tissue and bone marrow. They have an innate ability to self-renew and differentiate into multi-lineages, including osteoblasts, chondrocytes, myocytes and adipocytes (3-7). Recent studies have shown that MSCs have the unique ability to travel to injured tissue and actively participate in tissue repair $(8,9)$.

Interactions between MSCs and breast cancer cells were reported to modify distinct proliferative and morphological cancer cell changes $(10,11)$. More recently, an important study reported the interactions between cancer cells and hMSCs in tumor progression and metastasis by demonstrating that ovarian cancer-derived exosomes contributed to the generation of tumor-associated myofibroblasts from MSCs in tumor stroma (12).

Cancer cell invasion and metastasis is often facilitated by transdifferentiation through the epithelial-to-mesenchymal transition (EMT) (13). The EMT endows cancer cells with migratory, invasive and stem cell properties. At the molecular level, EMT is defined by downregulation of the epithelial differentiation marker E-cadherin and upregulation of mesenchymal markers such as Snail, N-cadherin, Twist, vimentin and $\beta$-catenin.

One of the main inducers of EMT in various cancers is TGF- $\beta$, which has been described to induce EMT in ovarian 
cancer (14). The intact TGF- $\beta$ signaling pathway appears to be necessary for metastatic phenotypes in an endometrial cancer model (15). In cancer, TGF- $\beta$ exerts the tumor promoter by enhancing cell invasion and metastasis in breast cancer (16). In addition to TGF- $\beta$, the production of growth factors, cytokines, chemokines, matrix-degrading enzymes and immunomodulatory mechanisms by MSCs augment tumor progression by providing a suitable environment (17).

EMT studies regarding the interactions between MSCs and tumor cells are insufficient for gynecologic cancer. In the present study, we investigate the growth pattern and potent inducer of EMT in gynecologic cancer cell lines (SKOV-3, IGROV-1 and Ishikawa) after co-culture with human MSCs (amnion, deciduas and bone marrow-derived). The co-cultured media were analyzed for 36 cytokines (C5a, CD40 ligand, G-CSF, GM-CSF, GRO $\alpha$, I-309, sICAM-1, IFN- $\gamma$, IL- $1 \alpha$, IL-1 $\beta$, IL-1 ra, IL-2, IL-4, IL-5, IL-6, IL-8, IL-10, IL-12p70, IL-13, IL-16, IL-17, IL-17E, IL-23, IL-27, IL-32 $\alpha$, IP-10, I-TAC, MCP-1, MIF, MIP-1 $\alpha$, MIP-1 $\beta$, serpin E1, RANTES, SDF-1, TNF $\alpha$ and STREM-1) to identify which expressed more cytokine than a monoculture of the cancer cell line. By using specific cytokines, we report whether a cytokine acts as a potent inducer of EMT using differentiation markers and morphologic changes. In addition, we analyze matrix metalloproteinases (MMP-2 and MMP-9) using western blot analysis. MMPs are well known as extracellular matrix degrading enzymes, and their activity is associated with tumor invasiveness and the migratory capacity of ovarian cancer cells (18). We also performed Matrigel invasion assay and wound healing assay for cell migration and invasion, respectively.

\section{Materials and methods}

Culture of cancer cell lines. The human ovarian cancer cell lines SKOV-3 and IGROV-1 were cultured in RPMI-1640 supplemented with heat inactivated $10 \%$ fetal bovine serum (FBS). The human endometrial cancer cell line Ishikawa was cultured in minimum essential medium (MEM) supplemented with 5\% FBS. All cells in the first passage were obtained from the American Type Culture Collection (ATCC; Manassas, VA, USA) and cultured in an incubator at $37^{\circ} \mathrm{C}, 5 \% \mathrm{CO}_{2}$.

Isolation and culture of human mesenchymal stem cells (hMSCs). The human mesenchymal stem cells (hMSCs) used originated from amnion, decidua and bone marrow. Bone marrow derived hMSCs in the first passage were obtained from the Department of Orthopedic Surgery of Guro Hospital. Placentas were obtained from clinically normal pregnant patients after vaginal or caesarean deliveries. The Institutional Review Board of Korea University Guro Hospital approved the procedure.

The amnion and decidua were mechanically peeled from the placenta and washed with phosphate-buffered saline (PBS) several times to remove blood. To collect mononuclear cells, the tissues were incubated with $1 \mathrm{mg} / \mathrm{ml}$ type I collagenase for $3 \mathrm{~h}$. After centrifugation, cells were washed with PBS and resuspended in $\alpha$-minimum essential medium ( $\alpha$-MEM; Invitrogen, Grand Island, NY, USA) supplemented with $10 \%$ FBS and $10 \mathrm{ng} / \mathrm{ml}$ basic fibroblast growth factor (bFGF). The cells were seeded into a T75 flask and cultures were main- tained at $37^{\circ} \mathrm{C}$ in a humidified atmosphere with $5 \% \mathrm{CO}_{2}$. The medium was replaced twice a week.

Direct co-culture. Primary hMSCs $\left(5 \times 10^{4}\right.$ cells $\left./ \mathrm{cm}^{2}\right)$ were seeded and allowed to adhere overnight. Cancer cell lines were then seeded at a density of $5 \times 10^{4}$ cells $/ \mathrm{cm}^{2}$ onto the monolayer of hMSCs. All cell types were cultured individually in parallel as controls. Cells were maintained in Dulbecco's modified Eagle's medium (DMEM; Invitrogen). After 7 days, the media was harvested and epithelial cells were retrieved as described below.

Human cytokine array. A cytokine array was performed in each conditioned medium after 3-4 days of culture (cancer cell line monoculture and co-culture with hMSCs). Human cytokine array panel A (R\&D Systems, Minneapolis, MN, USA) was used according to the manufacturer's instructions and analyzed 36 cytokines at a time. The 36 cytokines included C5a, CD40 ligand, G-CSF, GM-CSF, GRO $\alpha$, I-309, sICAM-1, IFN- $\gamma$, IL-1 $\alpha$, IL-1 $\beta$, IL-1ra, IL-2, IL-4, IL-5, IL-6, IL-8, IL-10, IL-12p70, IL-13, IL-16, IL-17, IL-17E, IL-23, IL-27, IL-32 $\alpha$, IP-10, I-TAC, MCP-1, MIF, MIP-1 $\alpha$, MIP-1 $\beta$, serpin E1, RANTES, SDF-1, TNF $\alpha$ and sTREM-1.

Treatment of TGF- $\beta 1$ and $I L-6$. In the human cytokine array, IL-6 was found to increase in all co-culture groups. For this reason, cancer cells were treated with IL-6 and EMT markers were checked. In addition, TGF- $\beta 1$ was added to each cancer cell line as a positive control. Each cancer cell line was treated with $10 \mathrm{ng} / \mathrm{ml}$ TGF- $\beta 1$ (Abcam, Milan, Italy) and $50 \mathrm{ng} / \mathrm{ml}$ IL-6 (Invitrogen) separately for 2 days. For all experiments, cells were cultured to $80 \%$ confluency and serum-starved for $24 \mathrm{~h}$ before treatment with TGF- $\beta 1$ and IL-6. Cell cultures were maintained at $37^{\circ} \mathrm{C}$ and $5 \% \mathrm{CO}_{2}$.

Total RNA isolation and reverse-transcriptase reaction. Total RNA extraction and purification were performed using RNeasy mini kit (Qiagen, Valencia, CA, USA) according to the manufacturer's protocol. Transcript amplification was performed using $1 \mu \mathrm{g} / \mu \mathrm{l}$ of total RNA and reverse transcriptase polymerase chain reaction using the SuperScript ${ }^{\mathrm{TM}}$ III First-Strand Synthesis System for RT-PCR kit (Invitrogen). The cycling conditions were $25^{\circ} \mathrm{C}$ for $10 \mathrm{~min}, 37^{\circ} \mathrm{C}$ for $120 \mathrm{~min}$ and $85^{\circ} \mathrm{C}$ for $5 \mathrm{~min}$.

Quantitative real-time PCR analysis. Real-time PCR was used to quantify E-cadherin, N-cadherin, Snail and Twist. Expression was normalized using the GAPDH housekeeping gene product as an endogenous reference. The primers and probes were designed for humans using Primer Express 2.0 (Applied Biosystems, Foster City, CA, USA). E-cadherin, $\mathrm{N}$-cadherin, Snail and Twist mRNA levels were quantified using TaqMan Real-Time PCR with an ABI 7300 system (Applied Biosystems). cDNAs were amplified by PCR using gene-specific probes and primer pairs as follows: E-cadherin (Assays-on-Demand, Hs01023894_m1; Applied Biosystems), N-cadherin (Hs00983056_m1), Snail (Hs00195591_m1) and Twist (Hs00361186_m1). The amplification conditions were $50^{\circ} \mathrm{C}$ for $2 \mathrm{~min}, 95^{\circ} \mathrm{C}$ for $10 \mathrm{~min}, 40$ cycles of amplication at $95^{\circ} \mathrm{C}$ for $15 \mathrm{sec}$, and $60^{\circ} \mathrm{C}$ for $1 \mathrm{~min}$. Data are presented as 
mean fold changes in gene expression relative to the control in 3 different experiments.

Western blot analysis. Total cell lysates of TGF- $\beta 1$ and IL-6 treated and untreated cancer cell lines were obtained by lysing the cells in RIPA buffer containing $50 \mathrm{mM}$ Tris- $\mathrm{HCl}, 150 \mathrm{mM}$ $\mathrm{NaCl}, 1 \%$ Triton $\mathrm{X}-100,0.1 \%$ SDS, $1 \%$ NaDeoxycholate (pH7.4), and protease inhibitor cocktail (1 mM phenylmethylsulfonyl fluoride, $10 \mu \mathrm{g} / \mathrm{ml}$ peptasin A, $10 \mu \mathrm{g} / \mathrm{ml}$ aprotinin and $5 \mu \mathrm{g} / \mathrm{ml}$ leupeptin).

Protein concentrations were measured using Bio-Rad protein assay kits (Bio-Rad Laboratories, Hercules, CA, USA). The protein lysates were then separated by SDS-PAGE and transferred onto nitrocellulose membrane (Hybond ${ }^{\mathrm{TM}}-\mathrm{P}$; Amersham Biosciences, Piscataway, NJ, USA). After blocking with PBS containing $0.2 \%$ Tween-20 and 5\% non-fat dry milk at $4^{\circ} \mathrm{C}$ overnight, membranes were incubated with the primary antibodies anti-E-cadherin (BD Biosciences, San Diego, CA, USA), anti-N-cadherin (Abcam, Cambridge, UK), anti-Snail (Abcam), anti-twist (Abcam), anti-MMP-2 (Cell Signaling Technology, Beverly, MA, USA) and anti-MMP-9 (Cell Signaling Technology). Protein detection was done with a chemiluminescence detection system (Pierce Chemical Co., Rockford, IL, USA).

Immunofluorescence. TGF- $\beta 1$ and IL- 6 treated and untreated cancer cell lines were fixed by treatment with $3.7 \%$ paraformaldehyde, permeabilized with $0.5 \%$ Triton X-100 for $10 \mathrm{~min}$, and blocked with $3 \%$ bovine serum albumin for $30 \mathrm{~min}$ at room temperature. After blocking, cells were incubated overnight at $4^{\circ} \mathrm{C}$ with primary antibodies, washed with PBS, incubated for $30 \mathrm{~min}$ at room temperature with Alexa Fluor-594 or -488-conjugated secondary antibody (Molecular Probes, Eugene, OR, USA), and co-stained with $2 \mu \mathrm{M}$ 4'6-diamidino2-phenylindole (DAPI) fluorescence (Molecular Probes) at $37^{\circ} \mathrm{C}$. After washing three times with PBS, the slides were mounted with Vectashield mounting medium (Vector Laboratories, Burlingame, CA, USA) and observed under confocal microscopy (LSM 700; Zeiss, Wetzlar, Germany).

Wound-healing assay. Cells were seeded at $1 \times 10^{5}$ cells/well in 12-well plates and preincubated for $24 \mathrm{~h}$ in serum-free RPMI (Invitrogen). A monolayer of cells at $90 \%$ confluence wounded with a plastic tip was allowed to close the wound in culture medium that was untreated, or treated with TGF- $\beta 1$ and IL-6. Cell migration into the wound surface was then monitored by microscopy after $24 \mathrm{~h}$ and reported as the estimated ratio of remaining wounded area relative to the initial wound area. Quantitation of monolayer closure was performed using the NIH Imaging program, and results are expressed as the percentage of wound closure. This assay was repeated three times independently.

Matrigel invasion assay. Cells (1×10\%/well) were seeded in the upper chamber, which was coated with Matrigel (Calbiochem, La Jolla, CA, USA) and serum-free medium containing different concentrations of the drugs was added to the lower chamber. After $48 \mathrm{~h}$ of incubation, non-migration cells were removed from the upper chamber with a cotton swab and the cells present on the lower surface of the insert were stained with Diff-Quick stain (Bio Chemical Sciences Inc. Science, Swedesboro, NJ, USA). Invading cells were then measured by microscopy. All experiments were repeated three times to confirm results.

Statistical analyses. A Student's t-test was performed to determine statistically significant differences between groups, and a P-value $<0.05$ was considered significant.

\section{Results}

Co-culture with hMSCs alters the proliferation of gynecologic cancer cells. Gynecologic cancer cell line cultures grew rapidly into cohesive monolayers on a plastic substratum. The cancer cells had typical cobblestone-like epithelial morphology. Amnion, decidua and bone marrow derived hMSCs formed a monolayer of homogeneous bipolar spindle-like cells with a whirlpool-like array. To investigate interactions between hMSCs and cancer cells, cancer cells were directly co-cultured with hMSCs (Fig. 1). IGROV-1 and Ishikawa in co-cultures were compared with IGROV-1 and Ishikawa in monocultures. Cancer cells in co-cultures with hMSCs had altered morphology and growth patterns in response to hMSCs. The cobblestone shaped cancer cell colony dissociated and a spindle-like, fibroblastic appearance took its place. The change was contact-dependent.

Cytokine expression in each co-cultivation. After analyzing for 36 cytokines, a distinction was found in the cytokine array between cancer cell monoculture and cancer cells cocultured with hMSCs. With each hMSC, SKOV-3 secreted GRO $\alpha$ (CXCL1), IL-6, IL-8 (CXCL8), MIF (GIF, DER6), MCP-1 (CCL2) and Serpin E1 (PAI-1). With each hMSC, IGROV-1 secreted GRO $\alpha$ (CXCL1), IL-6, IL-8 (CXCL8), MIF (GIF and DER6), MCP-1 (CCL2) and serpin E1 (PAI-1). With each hMSC, Ishikawa secreted GRO $\alpha$ (CXCL1), IL-1ra, IL-5, IL-6, MIF (GIF, DER6), MIP-1 $\beta$, serpinE1 and RANTES. In all cancer cell lines with hMSCs, only IL-6 increased more significantly than in monoculture (Fig. 2). This result shows that IL-6 was an inducer of EMT in the interactions of hMSCs and cancer cells.

TGF- $\beta 1$ and IL- 6 treatment induces mesenchymal morphologic changes associated with EMT. To investigate the role of IL-6 in promoting EMT, cancer cell lines were cultured with IL-6. In ddition, TGF- $\beta 1$ was added to each cancer cell line as a positive control. When TGF- $\beta 1$ was added to each cancer cell type, cell dissociation and fibroblastic morphologic changes were observed, as shown in EMT. A similar morphologic change occurred when IL- 6 was added. To study whether the shift in morphology occurred as a consequence of EMT, assays were performed by immunofluorescence for epithelial and mesenchymal markers. As shown in Fig. 3, untreated cancer cells strongly stained for the epithelial marker E-cadherin on the cell surface, while few stained for the mesenchymal marker Snail in the nucleus. In contrast, IL-6 and TGF- $\beta 1$ treated cancer cells had significantly increased Snail expression and decreased E-cadherin expression.

To determine whether changes in gene expression were detected at the protein level, western blot analysis was 


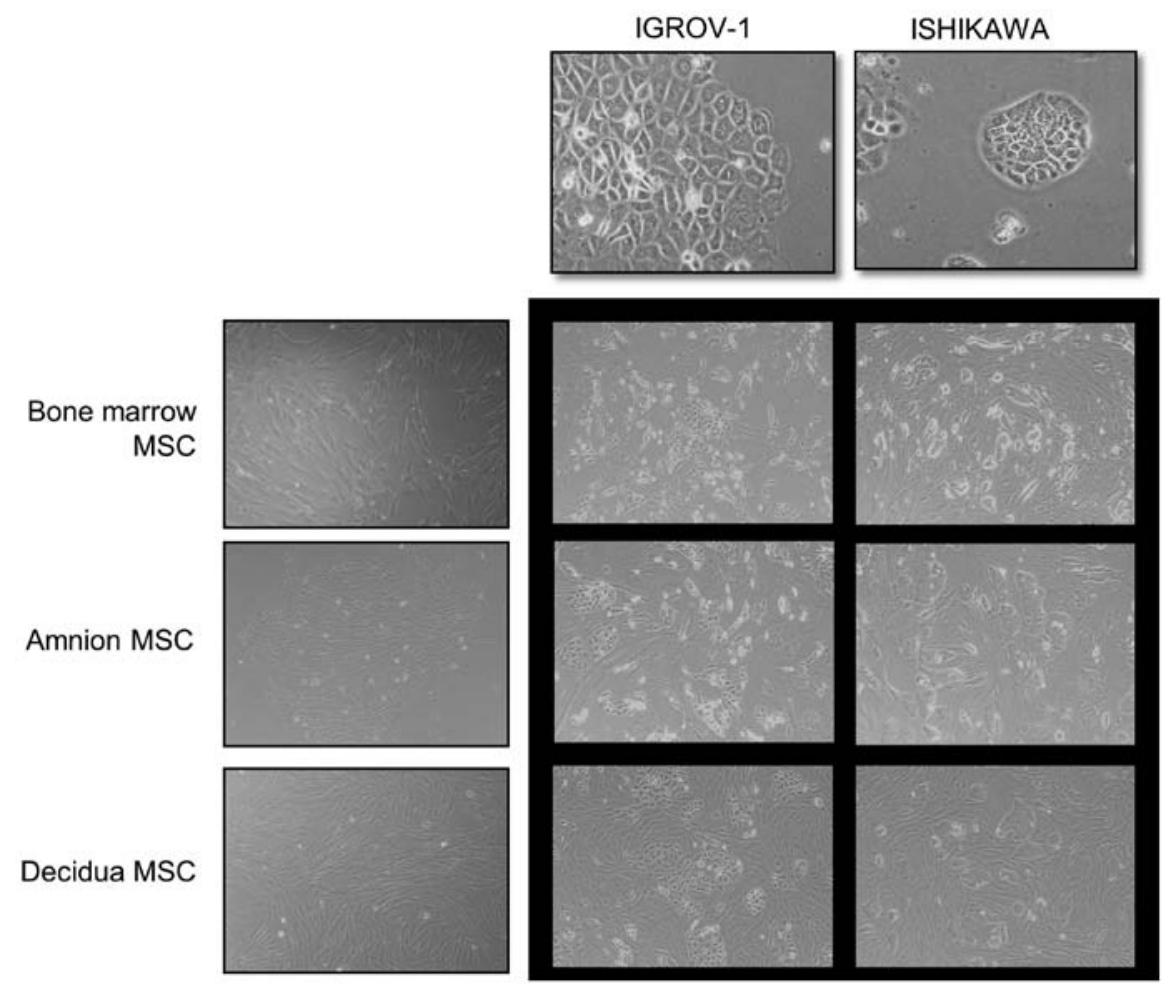

Figure 1. Co-culture with hMSCs alters the proliferation of gynecologic cancer cells. IGROV-1 and Ishikawa cells were co-cultured with bone marrow, amnion and decidua-derived MSCs (Black box). Cell proliferation and growth were altered in co-cultures with all hMSCs. Cancer cells in co-cultures with hMSCs had altered morphology and growth patterns in response to hMSCs. The cobblestone shaped cancer cell colony dissociated and became spindle-like and fibroblastic in appearance. Images were assessed by phase contrast microscope. (Original magnification, x100).
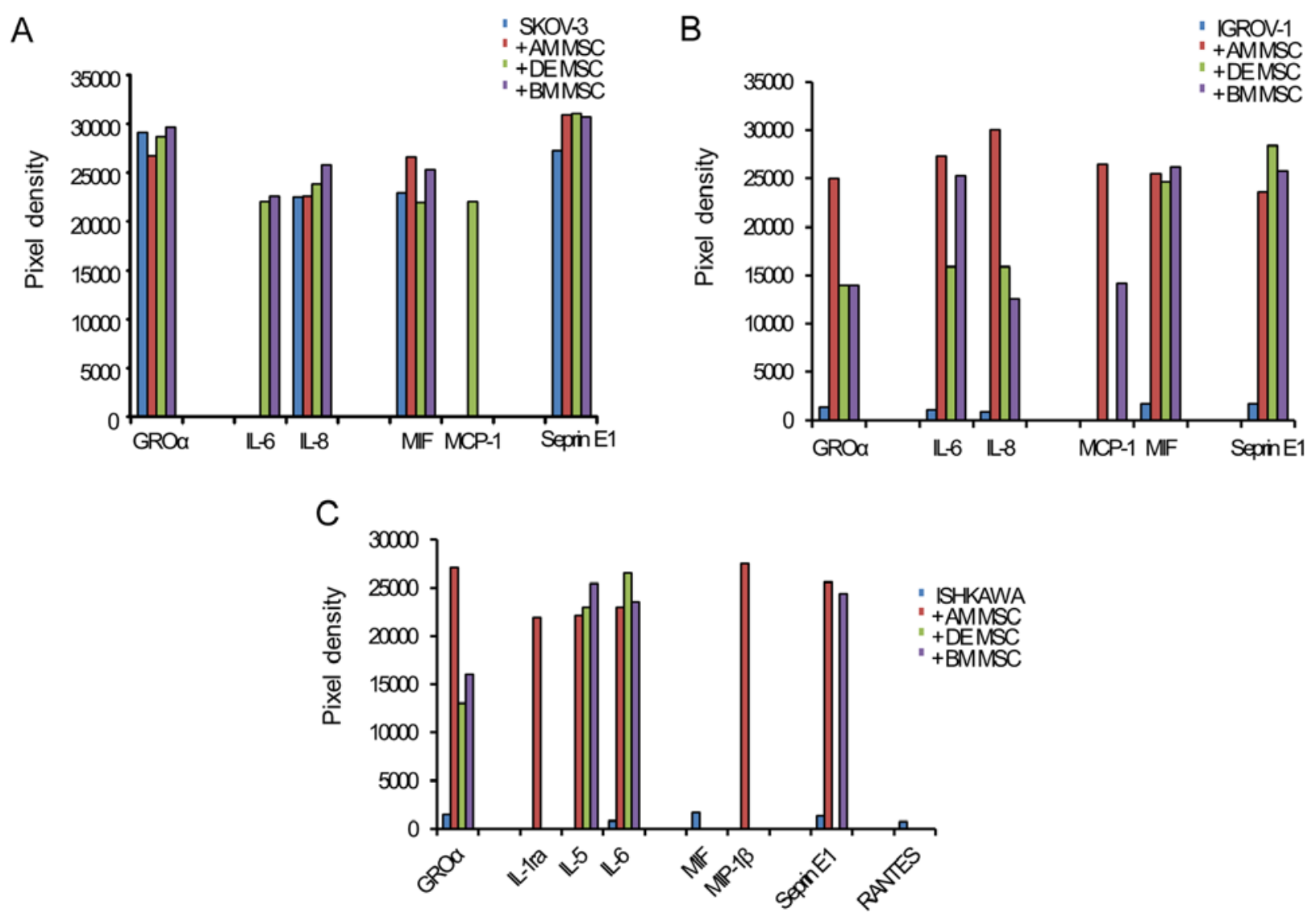

Figure 2. Expression of cytokines in co-cultivation. Cytokine optical density was quantified using Image-Pro Plus v.5.1 software. (A) SKOV-3, (B) IGROV-1, (C) Ishikawa. BM MSC, bone marrow-derived MSC; AM MSC, amnion-derived MSC; DE MSC, decidua-derived MSCs. In all cancer cell lines, IL-6 increased in co-culture more than in monoculture. 

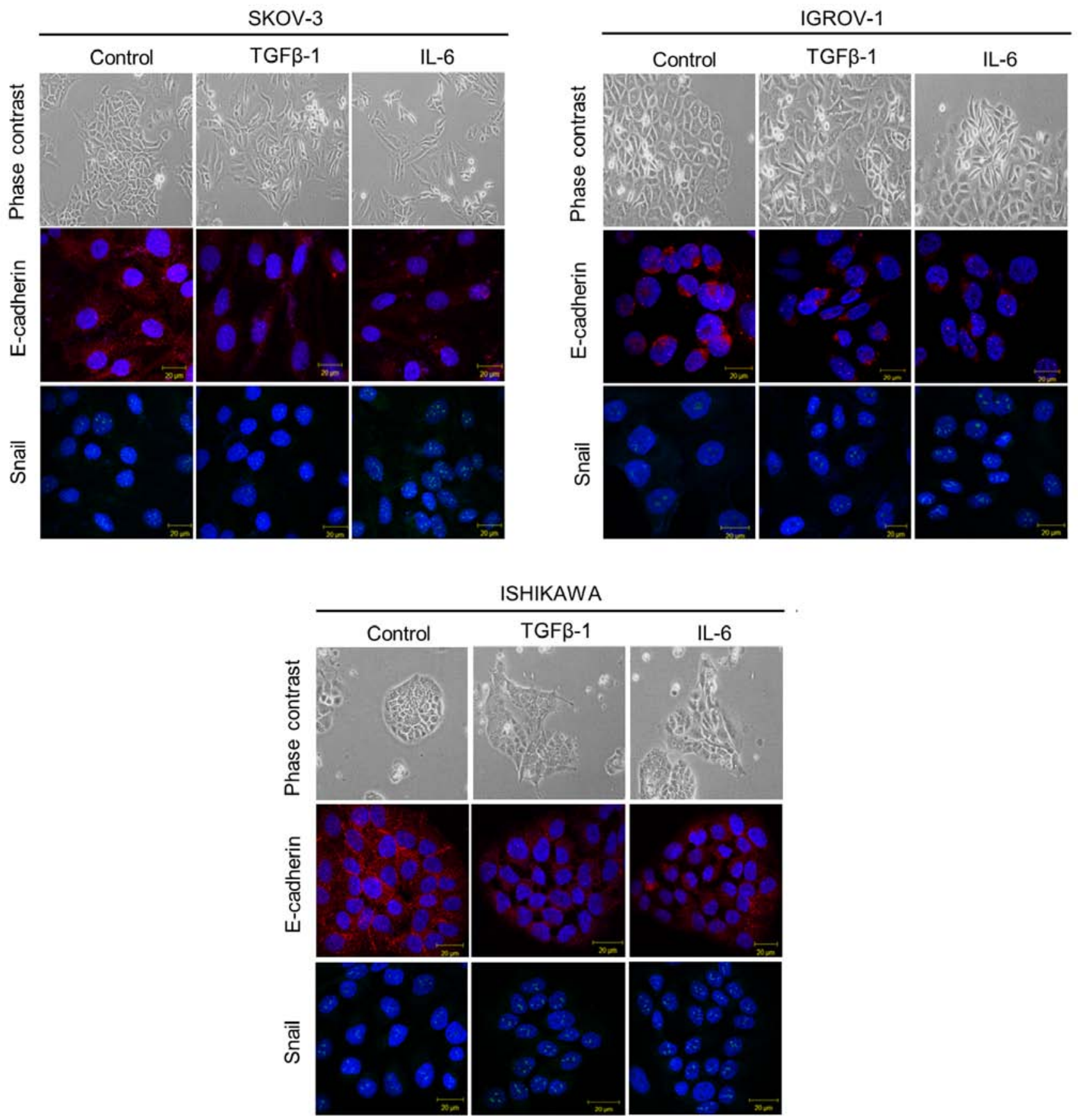

Figure 3. TGF- $\beta 1$ and IL-6 treatment induced mesenchymal morphologic changes associated with EMT markers. Cells were treated with TGF- $\beta 1$ (10 ng/ml) and IL-6 $(50 \mathrm{ng} / \mathrm{ml})$ separately for 48-72 h. Phenotype differences between the untreated and treated group were visualized by phase-contrast microscopy. Cells were stained with anti-E-cadherin plus 594-conjugated secondary antibody (red) and anti-snail plus FITC-conjugated secondary antibody (green). Cells were visualized through fluorescence microscopy. Samples were co-stained with DAPI. Untreated cancer cells strongly stained for the epithelial marker E-cadherin on the cell surface, and few stained for the mesenchymal marker Snail in the nucleus. In contrast, IL- 6 and TGF- $\beta 1$ treated cancer cells had significantly increased Snail expression and decreased E-cadherin expression. (Original magnification, $\mathrm{x} 400$ ).

performed (Fig. 4A). In SKOV-3 cultured with TGF- $\beta 1$, E-cadherin expression decreased while $\mathrm{N}$-cadherin and Snail expression increased. When IL-6 was added to SKOV-3, similar changes in E-cadherin, $\mathrm{N}$-cadherin and Snail levels were observed. Decreased E-cadherin and increased Snail were also observed in each TGF- $\beta 1$ and IL- 6 treated IGROV-1. In Ishikawa, Snail increased while E-cadherin decreased in both treated groups.

In quantitative real-time PCR, there were significant changes in EMT markers in both TGF- $\beta 1$ and IL-6 treat- ment groups (Fig. 4B). When TGF- $\beta 1$ was added to SKOV-3, E-cadherin decreased while mesenchymal markers such as $\mathrm{N}$-cadherin and Snail significantly increased. With IL-6, significant decreases in E-cadherin and increases in Snail were noted. In IGROV-1, a significant increase in Twist, a mesenchymal marker, was observed in both the TGF- $\beta 1$ and IL-6 treatment groups. When TGF- $\beta 1$ was added to Ishikawa, significant increases in Snail and Twist were detected. Similarly, significant increases in N-cadherin, Snail and Twist were noted in Ishikawa with IL-6. 
A
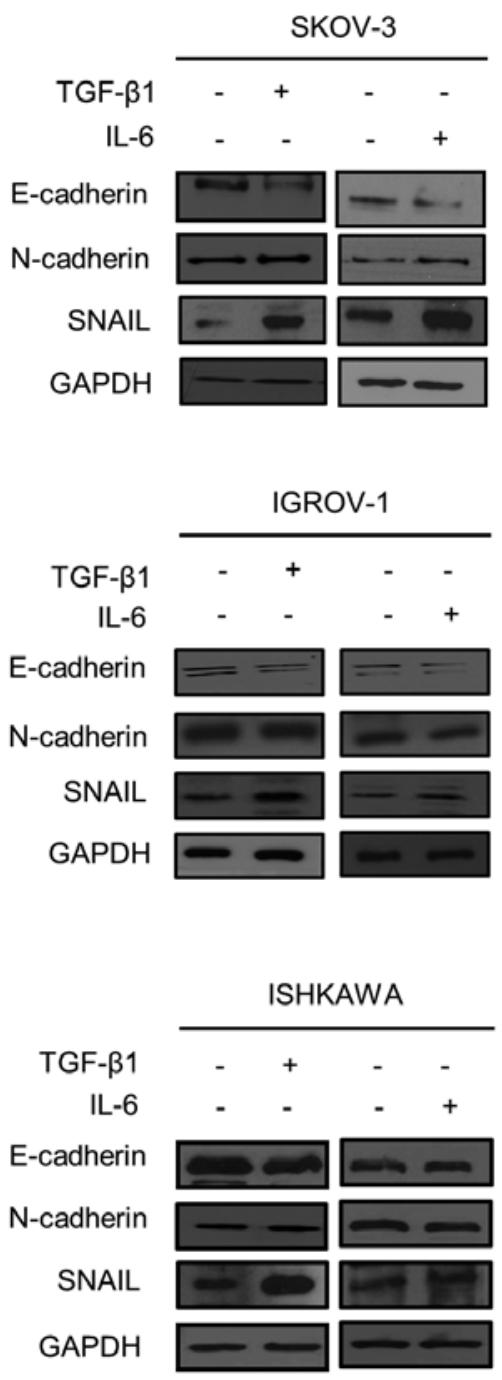

B
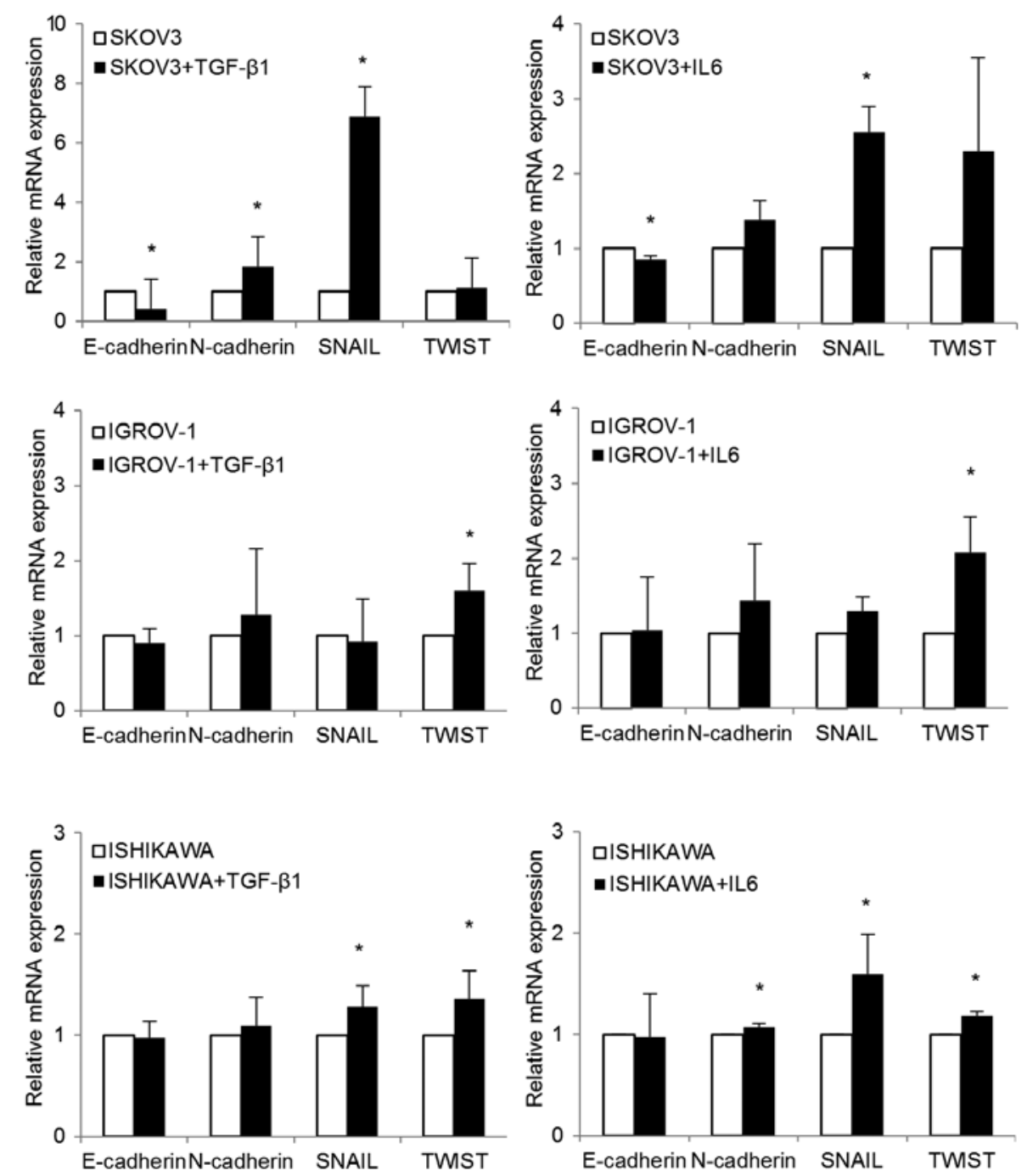

Figure 4. EMT markers after TGF- $\beta 1$ and IL-6 treatment. (A) Cells treated with TGF- $\beta 1$ (10 ng/ml) and IL-6 (50 ng/ml) for 48-72 h. Western blotting was performed with anti-E-cadherin, anti-N-cadherin or anti-Snail. In SKOV-3 cultured with TGF- $\beta 1$, E-cadherin expression decreased while N-cadherin and Snail expression increased noticeably. When IL-6 was added to SKOV-3, similar changes in E-cadherin, N-cadherin and Snail levels were observed. Decreased E-cadherin and increased Snail were also observed in TGF- $\beta 1$ and IL-6 treated IGROV-1. Snail increased in Ishikawa while E-cadherin decreased in both treated groups. (B) Cells treated with TGF- $\beta 1$ and IL-6 for $48 \mathrm{~h}$ were collected, and RNA was subjected to quantitative real-time PCR using specific primers for E-cadherin, N-cadherin and Snail. Relative mRNA levels were normalized to corresponding GAPDH mRNA expression. Bars represent the standard deviation of three independent experiments conducted in triplicate. When TGF- $\beta 1$ was added to SKOV-3, E-cadherin decreased while N-cadherin and Snail significantly increased. For IL-6, significant decreases in E-cadherin and increases in Snail were noted. In IGROV-1, a significant increase in Twist was observed in both the TGF- $\beta 1$ and IL-6 treatment groups. When TGF- $\beta 1$ was added to Ishikawa, significant increases in Snail and Twist were found. Similarly, significant increases in N-cadherin, Snail, and Twist were noted in Ishikawa with IL-6. ${ }^{*} \mathrm{P}<0.05$, compared to cells treated and control.

TGF- $\beta 1$ and IL- 6 treatment promotes metastatic potential of gynecologic cancer cells by EMT. Cell migration and invasion were evaluated by analyzing the overexpression of MMPs. Western blot analysis showed that MMP-2 and MMP-9 expression increased in each TGF- $\beta 1$ and IL-6 treated cell line (Fig. 5A). Another important aspect of EMT is an increase in cellular motility. Separate treatment with TGF- $\beta 1$ and IL- 6 significantly enhanced the migration ability of cancer cells compared to untreated cells $(\mathrm{P}<0.05$; Fig. 5B). Matrigel invasion assay showed that IL- 6 and TGF- $\beta 1$ were both effective at increasing cancer cell invasion. IL- 6 and TGF- $\beta 1$ treated cancer cells displayed significantly increased invasiveness compared to untreated cancer cells $(\mathrm{P}<0.05$; Fig. 5C).

\section{Discussion}

Developing tumors recruit MSCs through releasing endocrine and paracrine signals (19). MSCs are multipotent and have the ability to preferentially migrate towards the tumor microenvironment. Several reports have implicated MSCs in promoting tumor growth and metastasis $(13,20,21)$. Increasing evidence suggests that dynamic interaction between cancer cells and their microenvironment supports tumor growth and 
A

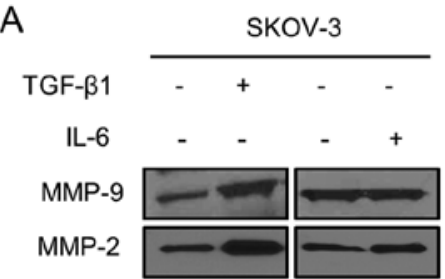

B

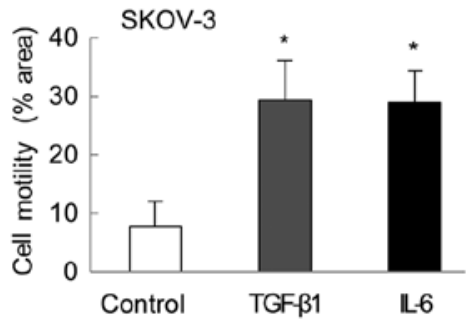

C
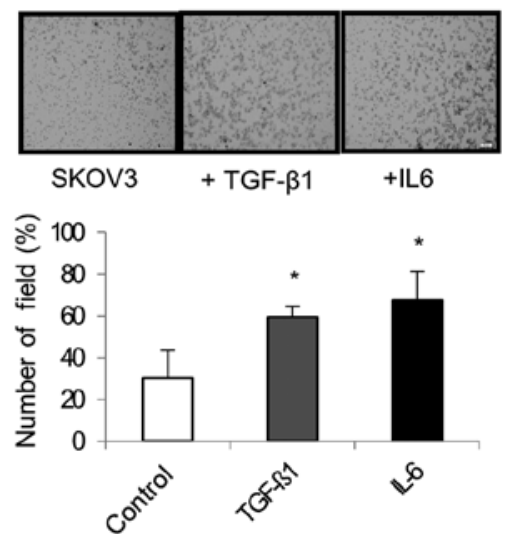
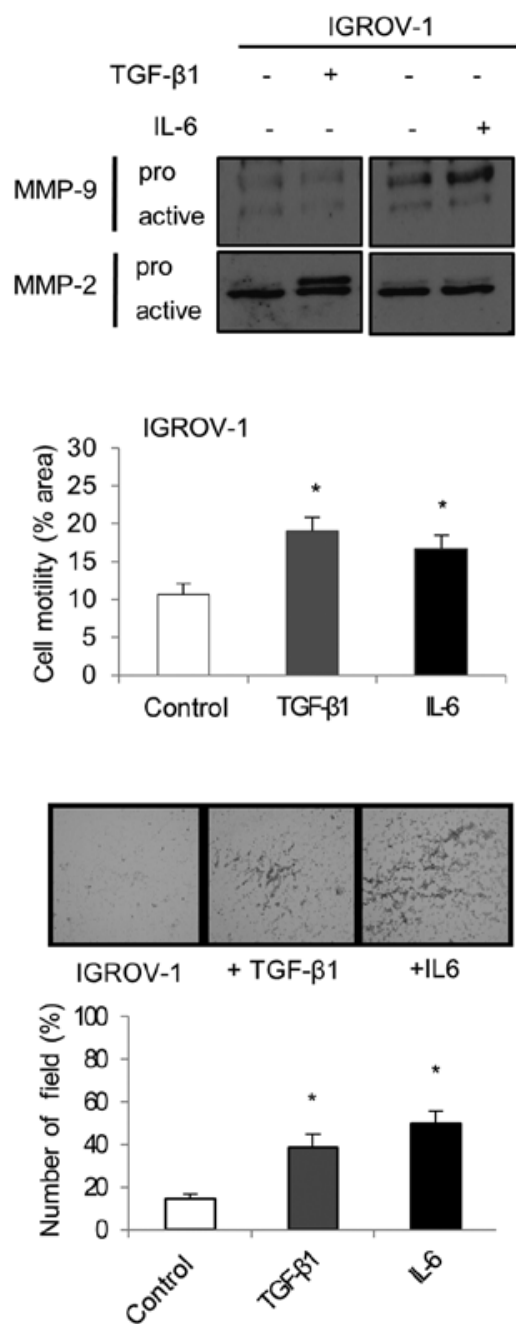

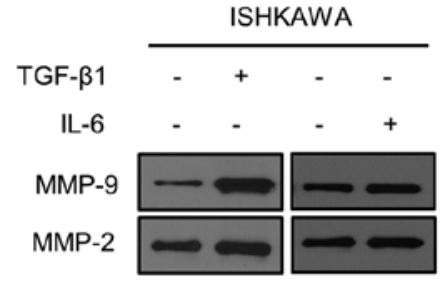

ISHIKAWA
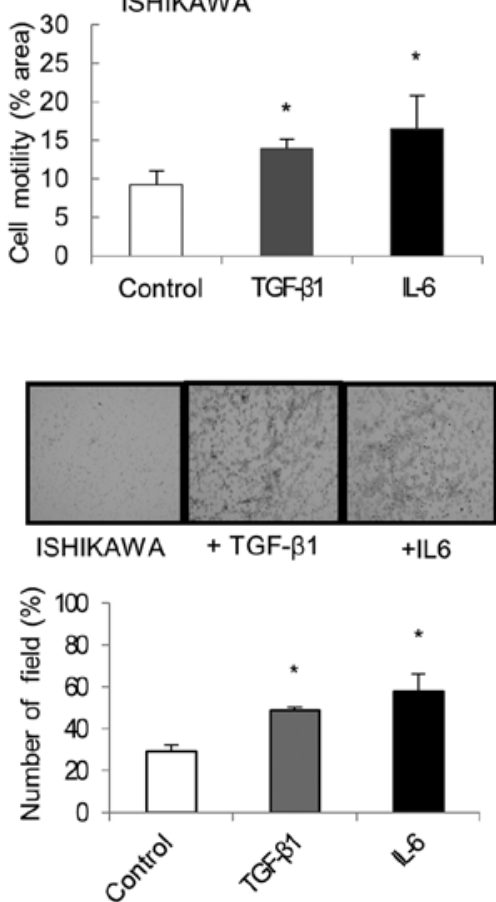

Figure 5. Effect of TGF- $\beta 1$ and IL- 6 on MMP2/9 expression in gynecologic cancer cell lines. TGF- $\beta 1$ and IL-6 treatment promotes metastatic potential of gynecologic cancer cells. Cells treated with TGF- $\beta 1(10 \mathrm{ng} / \mathrm{ml})$ and IL-6 $(50 \mathrm{ng} / \mathrm{ml})$ for $48-72 \mathrm{~h}$. (A) The conditioned culture media of samples was subjected to western blotting using antibodies against MMP-2 and MMP-9. Western blot analysis showed that MMP-2 and MMP-9 expression increased in each TGF- $\beta 1$ and IL-6 treated cell line. (B and C) The migration and invasive ability of the cells were evaluated by wound healing (B) and the Matrigel invasion assay (C) after 24 h. IL- 6 and TGF- $\beta 1$ treated cancer cells displayed significantly increased invasiveness and migration compared to untreated cancer cells. Data are expressed as means of three independent experiments.

metastasis. However, the role of MSCs in gynecologic tumor microenvironment and the underlying mechanisms remain unclear. The present study was performed to better understand these interactions.

Notably, MSCs have been reported to promote breast cancer metastasis by stimulating EMT (2). EMT is a complex morphogenetic process in which epithelial cells lose their innate characteristics and gain new mesenchymal properties. It is an important biological process responsible for cancer cell invasion and metastasis. In addition, this morphogenetic process is commonly observed in gynecologic cancer patients $(14,22,23)$.

In these experiments, we showed that gynecologic cancer cells co-cultured with hMSCs had altered morphology and growth patterns in response to hMSCs. The compact cancer cell colony organization dissociated and transformed into fibroblastic spindle-shaped appearances. This change was contact-dependent and similar to that of a breast cancer model (2). MSCs are thought to affect cancer cell growth and differentiation.
Various signaling pathways that are associated with cytokines and growth factors in the tumor microenvionment trigger the EMT process (24). This happens by activating variable signaling pathways and their target genes (25). For example TGF- $\beta$, and growth factors such as hepatocyte growth factor (HGF), epidermal growth factor (EGF), and fibroblast growth factors (FGFs) can induce EMT in vitro after activating their receptors in specific cell types (26). The TGF- $\beta$ family is one of the best characterized EMT inducers in cancer pathogenesis. TGF- $\beta$ is a potent regulator of EMT and cell stemness in breast cancer stem cells (16).

TGF- $\beta 1$ and interleukin-like EMT inducer independently promote an EMT phenotype in mouse mammary epithelial cells (27). This was confirmed in our experiment. In the gynecologic cancer cell lines (SKOV-3, IGROV-1 and Ishikawa), IL-6 increased in co-culture with hMSCs more than in monoculture. Interactions between hMSCs and cancer cells released IL-6 into the tumor microenvironment. On the basis of this result, we focused on IL- 6 and further investigated whether IL- 6 could be an inducer of EMT. This was done using a 
number of specific EMT differentiation markers and morphologic changes.

After each TGF- $\beta 1$ and IL-6 treatment of gynecologic cancer cell lines, both groups appeared to have morphological changes similar to those of mesenchymal cells. The cancer cell colony dissociated into spindle-shaped cells like those in the EMT process. We also observed that IL-6 and TGF- $\beta 1$ treated cancer cells significantly increased Snail expression and decreased E-cadherin expression using immunofluorescence. IL-6 seems to mediate the EMT process by decreasing epithelial markers and increasing mesenchymal markers.

EMT differentiation markers were also examined after IL- 6 treatment by quantitative RT-PCR and western blot analysis. In agreement with previous reports on other cancers $(27,28)$, we showed that IL-6 induced EMT in gynecologic cancer cell lines by acquiring a mesenchymal morphology with increased expression of mesenchymal markers. However, there were differences between each cancer cell line in the expression of EMT markers. SKOV-3 with IL-6 had significantly decreased E-cadherin and increased Snail on RT-PCR, with decreased E-cadherin and increased Snail and N-cadherin on western blot analysis. IGROV-1 with IL-6 had noticeably increased Twist on RT-PCT, with decreased E-cadherin and increased Snail on western blot analysis. Ishikawa with IL-6 had significantly increased Snail, N-cadherin and Twist on RT-PCT, with decreased E-cadherin and increased Snail on western blot analysis.

Cancer cells underwent EMT in response to IL-6 and TGF- $\beta 1$, which enhanced invasion and metastasis. We observed that IL-6 induces the release of matrix metalloproteinases (MMPs) from gynecologic cancer cell lines (SKOV-3, IGROV-1 and Ishikawa). Matrix metalloproteinases (MMPs) are zinc-dependent endopeptidases that cleave extracellular matrix (ECM) molecules. MMP-2 and MMP-9 are expressed by epithelial cells, particularly cancer cells, and can degrade type IV collagen of basement membranes (29). The degree of enzymatic degradation of basement membrane type IV collagen may correlate with the metastatic potential and modulating tumor invasion of carcinoma $(30,31)$. High MMP-2/MMP-9 expression was associated with metastatic potential in several human carcinomas.

IL-6 is a pleiotropic cytokine involved in the acute phase of inflammation (32). The tumor-promoting action of IL-6 has been shown in experimental cancer models. In ovarian cancer, IL-6 enhances tumor cell proliferation and increases resistance to chemotherapy via the JAK/STAT signaling pathway (33). IL-6 and its receptor are also overexpressed in malignant ascites from ovarian cancer and correlate with poor prognosis (28). The endogenous expression of IL-6 by MSCs increased breast cancer cell growth and metastasis (34). MSCs stimulated tumor growth through the paracrine production of secreted IL-6. In breast cancer cells co-cultured with MSCs, paracrine IL-6 was found to be the principal mediator of STAT3 phosphorylation. STAT3 phosphorylation was lost when IL-6 was depleted from MSC conditioned media or when the IL-6 receptor was blocked in tumor cells (35).

The present study may confirm that expression of interleukin- 6 by interactions between gynecologic cancer cells and hMSCs induces EMT. Our results suggest that IL-6 plays a critical role in oncogenic EMT mediated by the interaction of hMSCs and cancer cells. IL-6 can serve as a key regulator of invasion and metastasis in gynecologic cancer. Future studies should address the role of IL-6 in mechanotransduction, which modulates changes in gene expression to alter gynecologic cancer cell differentiation and metastatic potential.

\section{Acknowledgements}

The present study was supported by the National Research Foundation of Korea (NRF) grant funded by the Korea government (MEST) (no. 2010-0021906).

\section{References}

1. Swartz MA, Iida N, Roberts EW, Sangaletti S, Wong MH, Yull FE, Coussens LM and DeClerck YA: Tumor microenvironment complexity: Emerging roles in cancer therapy. Cancer Res 72: 2473-2480, 2012.

2. Martin FT, Dwyer RM, Kelly J, Khan S, Murphy JM, Curran C, Miller N, Hennessy E, Dockery P, Barry FP, et al: Potential role of mesenchymal stem cells (MSCs) in the breast tumour microenvironment: Stimulation of epithelial to mesenchymal transition (EMT). Breast Cancer Res Treat 124: 317-326, 2010.

3. Lu LL, Liu YJ, Yang SG, Zhao QJ, Wang X, Gong W, Han ZB, $\mathrm{Xu} \mathrm{ZS}, \mathrm{Lu} \mathrm{YX}$, Liu D, et al: Isolation and characterization of human umbilical cord mesenchymal stem cells with hematopoiesis-supportive function and other potentials. Haematologica 91: 1017-1026, 2006.

4. Prockop DJ, Sekiya I and Colter DC: Isolation and characterization of rapidly self-renewing stem cells from cultures of human marrow stromal cells. Cytotherapy 3: 393-396, 2001.

5. In 't Anker PS, Scherjon SA, Kleijburg-van der Keur C, de GrootSwings GM, Claas FH, Fibbe WE and Kanhai HH: Isolation of mesenchymal stem cells of fetal or maternal origin from human placenta. Stem Cells 22: 1338-1345, 2004.

6. Pittenger MF, Mackay AM, Beck SC, Jaiswal RK, Douglas R, Mosca JD, Moorman MA, Simonetti DW, Craig S and Marshak DR: Multilineage potential of adult human mesenchymal stem cells. Science 284: 143-147, 1999.

7. Deans RJ and Moseley AB: Mesenchymal stem cells: Biology and potential clinical uses. Exp Hematol 28: 875-884, 2000.

8. Chen J, Zhang ZG, Li Y, Wang L, Xu YX, Gautam SC, Lu M, Zhu Z and Chopp M: Intravenous administration of human bone marrow stromal cells induces angiogenesis in the ischemic boundary zone after stroke in rats. Circ Res 92: 692-699, 2003.

9. Spaeth E, Klopp A, Dembinski J, Andreeff M and Marini F: Inflammation and tumor microenvironments: Defining the migratory itinerary of mesenchymal stem cells. Gene Ther 15 : 730-738, 2008.

10. Hombauer $\mathrm{H}$ and Minguell JJ: Selective interactions between epithelial tumour cells and bone marrow mesenchymal stem cells. Br J Cancer 82: 1290-1296, 2000.

11. Fierro FA, Sierralta WD, Epuñan MJ and Minguell JJ: Marrowderived mesenchymal stem cells: Role in epithelial tumor cell determination. Clin Exp Metastasis 21: 313-319, 2004.

12. Cho JA, Park H, Lim EH, Kim KH, Choi JS, Lee JH, Shin JW and Lee KW: Exosomes from ovarian cancer cells induce adipose tissue-derived mesenchymal stem cells to acquire the physical and functional characteristics of tumor-supporting myofibroblasts. Gynecol Oncol 123: 379-386, 2011.

13. Karnoub AE, Dash AB, Vo AP, Sullivan A, Brooks MW, Bell GW, Richardson AL, Polyak K, Tubo R and Weinberg RA: Mesenchymal stem cells within tumour stroma promote breast cancer metastasis. Nature 449: 557-563, 2007.

14. Vergara D, Merlot B, Lucot JP, Collinet P, Vinatier D, Fournier I and Salzet M: Epithelial-mesenchymal transition in ovarian cancer. Cancer Lett 291: 59-66, 2010.

15. Lei X, Wang L, Yang J and Sun LZ: TGFbeta signaling supports survival and metastasis of endometrial cancer cells. Cancer Manag Res 2009: 15-24, 2009.

16. Imamura $T$, Hikita $A$ and Inoue $Y$ : The roles of TGF- $\beta$ signaling in carcinogenesis and breast cancer metastasis. Breast Cancer 19: 118-124, 2012. 
17. Spaeth EL, Dembinski JL, Sasser AK, Watson K, Klopp A, Hall B, Andreeff M and Marini F: Mesenchymal stem cell transition to tumor-associated fibroblasts contributes to fibrovascular network expansion and tumor progression. PLoS One 4: e4992, 2009.

18. Do TV, Kubba LA, Du H, Sturgis CD and Woodruff TK: Transforming growth factor-beta1, transforming growth factorbeta2, and transforming growth factor-beta3 enhance ovarian cancer metastatic potential by inducing a Smad3-dependent epithelial-to-mesenchymal transition. Mol Cancer Res 6 : 695-705, 2008

19. Stagg J: Mesenchymal stem cells in cancer. Stem Cell Rev 4: $119-124,2008$

20. Shinagawa K, Kitadai Y, Tanaka M, Sumida T, Kodama M, Higashi Y, Tanaka S, Yasui W and Chayama K: Mesenchymal stem cells enhance growth and metastasis of colon cancer. Int J Cancer 127: 2323-2333, 2010.

21. Albarenque SM, Zwacka RM and Mohr A: Both human and mouse mesenchymal stem cells promote breast cancer metastasis. Stem Cell Res (Amst) 7: 163-171, 2011.

22. Lee MY and Shen MR: Epithelial-mesenchymal transition in cervical carcinoma. Am J Transl Res 4: 1-13, 2012.

23. Castilla MÁ, Moreno-Bueno G, Romero-Pérez L, Van De Vijver K, Biscuola M, López-García MÁ, Prat J, Matías-Guiu X, Cano A, Oliva E, et al: Micro-RNA signature of the epithelialmesenchymal transition in endometrial carcinosarcoma. J Pathol 223: 72-80, 2011.

24. Francí C, Takkunen M, Dave N, Alameda F, Gómez S, Rodríguez R, Escrivà M, Montserrat-Sentís $\mathrm{B}$, Baró $\mathrm{T}$, Garrido M, et al: Expression of Snail protein in tumor-stroma interface. Oncogene 25: 5134-5144, 2006.

25. Hogan NM, Dwyer RM, Joyce MR and Kerin MJ: Mesenchymal stem cells in the colorectal tumor microenvironment: Recent progress and implications. Int J Cancer 131: 1-7, 2012.
26. Morel AP, Lièvre M, Thomas C, Hinkal G, Ansieau S and Puisieux A: Generation of breast cancer stem cells through epithelial-mesenchymal transition. PLoS One 3: e2888, 2008.

27. Sullivan NJ, Sasser AK, Axel AE, Vesuna F, Raman V, Ramirez N, Oberyszyn TM and Hall BM: Interleukin-6 induces an epithelial-mesenchymal transition phenotype in human breast cancer cells. Oncogene 28: 2940-2947, 2009.

28. Yadav A, Kumar B, Datta J, Teknos TN and Kumar P: IL-6 promotes head and neck tumor metastasis by inducing epithelialmesenchymal transition via the JAK-STAT3-SNAIL signaling pathway. Mol Cancer Res 9: 1658-1667, 2011.

29. Chen P and Parks WC: Role of matrix metalloproteinases in epithelial migration. J Cell Biochem 108: 1233-1243, 2009.

30. Zeng ZS, Cohen AM and Guillem JG: Loss of basement membrane type IV collagen is associated with increased expression of metalloproteinases 2 and 9 (MMP-2 and MMP-9) during human colorectal tumorigenesis. Carcinogenesis 20: 749-755, 1999.

31. Thomas GT, Lewis MP and Speight PM: Matrix metalloproteinases and oral cancer. Oral Oncol 35: 227-233, 1999.

32. Kishimoto T: Interleukin-6: Discovery of a pleiotropic cytokine. Arthritis Res Ther 8 (Suppl 2): S2, 2006.

33. Duan Z, Foster R, Bell DA, Mahoney J, Wolak K, Vaidya A, Hampel C, Lee H and Seiden MV: Signal transducers and activators of transcription 3 pathway activation in drug-resistant ovarian cancer. Clin Cancer Res 12: 5055-5063, 2006.

34. Shah K: Mesenchymal stem cells engineered for cancer therapy. Adv Drug Deliv Rev 64: 739-748, 2012.

35. Sasser AK, Sullivan NJ, Studebaker AW, Hendey LF, Axel AE and Hall BM: Interleukin-6 is a potent grow th factor for ER-alpha-positive human breast cancer. FASEB J 21: 3763-3770, 2007. 